I.A.A.R.C.

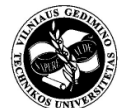

Institute of Internet and Intelligent Technologies

Vilnius Gediminas Technical University

Saulètekio al. 11, 10223 Vilnius, Lithuania

http://www.isarc2008.vgtu.lt/
The $25^{\text {th }}$ International Symposium on Automation and Robotics in Construction

June 26-29, 2008

ISARC-2008

\title{
TELEMETRIC SYSTEM FOR MANAGEMENT IN CONCRETE PROCESS
}

\author{
Andrzej Karłowski \\ Poznań University off Technology \\ andrzej.karlowski@ikb.poznan.pl
}

\begin{abstract}
In day of Internet and mobile phones of wireless broadcast of data, GPS - tools to collecting and transmitting of information, in real time one should to create tools, which will assemble this information and will be capable to aid technological decision in constriction industry. Building and first of all management should use this tools in larger range.

System TSforMinCP be leaning on the newest telecommunicational solutions it - uses: the wireless net GSM/GPRS/APN, internet (the server of www and side of web) to measurement of temperature, wind and moisture in initial period of maturation of concrete. We on square of building in concreted element place the sensors of temperature from transmitter the GSM.
\end{abstract}

\section{KEYWORDS}

GSM, GPRS, decision making, decision analysis

\section{INTRODUCTION}

The problem of the thermal influence of concrete hardening in the beginning phase of concrete setting has a great influence on the increase of internal stress and the formation of cracks $[1,2]$. Despite many years devoted to research this subject is being still developed and improved. However, on account of different dimensions of elements from concrete as well as different time of their preparation and above all the applied types of concrete the most reliable results are achieved on site. In the age of Internet, mobile phones and wireless data transmission: GPS and GSM the use of these popular and easily accessible communications devices in the management of building processes seems essential. These devices allow to communicate in a real time which considerably facilitates quick decision making and the management of building processes.

Only several years ago the rapid development of mobile communications was stimulated exclusively by technologies that used voice transmission and sending SMS messages. Wireless solutions based on radio modems and leased cable analogue connections used to serve as the only solution to send data at that time. These two solutions were marked by the high price of the system construction and expensive connections maintenance. However, 
the situation has changed since the introduction of GPRS service (General Packet Radio Services) that enables package data transmission as in Ethernet network in standard GSM environment.

The system that is the subject of this article is based on wireless communication which uses GSM telephony to send data from the recorded located on site to the database and www servers. Modus operandi of the system consists in monitoring the temperature in the beginning period of settingmaturing of concrete by temperature detectors located in concrete and outside - in its surroundings. Then the data on current temperatures on separate detectors are sent by radio via GSM network in the form of SMS messages to people supervising the process of the concrete setting and above all in the form of GPRS data via communications module to database and www servers.

\section{GPRS AND APN NETWORK -GLOBAL COMMUNICATION}

GSM network in APN standard is used to send data, the network uses any operator so the communication may take place in any area provided that there is the telecommunications infrastructure which enables the calls in GSM standard. Measuring data recorded on a recorder are sent via GSM modem as SMS message to people supervising the process of ripening of concrete and as GPRS data to communication module [3]. The APN standard (Access point name) ensures the organized exchange of data between recorders and communications module since each telemetric module has its own unique IP address [4]. Communications module with the use of appropriate software sends data to either databases or www server.

\section{COMMUNICATION INTERFACE}

Data registered by telemetric module are sent via GSM network to communications module which communicates with the database and www servers via the appropriate software. The communications software for the operation of recorders and communicators with GPRS modem offers the user great possibilities for data processing. Communications driver operates in Ms Windows platforms, makes the current data available via a standard OPC interface (OLE for Process Control) and archival data - via CSV/XML files. It is also possible to record the measuring data directly via ODBC interface to a standard relational database (e.g. MS SQL Server, MSDE, Oracle). This solution gives a wide range of possibilities and enables a direct connection with modern systems such as: visualization (in the form of graphs as well as animation of temperature variation in time), spreadsheets and data processing systems (as analytical, optimizing and diagnostic tools)

Depending on configuration settings independent for each measuring telemetric module it is possible to communicate via questioning the module every specified period of time - according to a determined schedule, at any time on demand and during the alarms - the exceeding of minimum or maximum temperature.

\section{GATHERING AND USING THE GATHERED DATA}

To the registered in this way in the database task shall be assigned measuring data stored according to a determined pattern - model that ensures appropriate selection of the results. Data gathered and registered in the database are assigned according to the determined pattern to a specific site and concern the specific element having its specific geometric size and being prepared in a specific period of time in accordance with the specific prescription and the class of the concrete. The measuring data gathered in this way and additional information form the knowledge base which can be used to optimization and simulation of concrete ripening processes as well as the choice of the appropriate concrete mixture depending on the size of the element, the required class of concrete, season and atmospheric conditions in order to acquire the designed concrete.

The results of the conducted research were presented on Figures (1-5). 


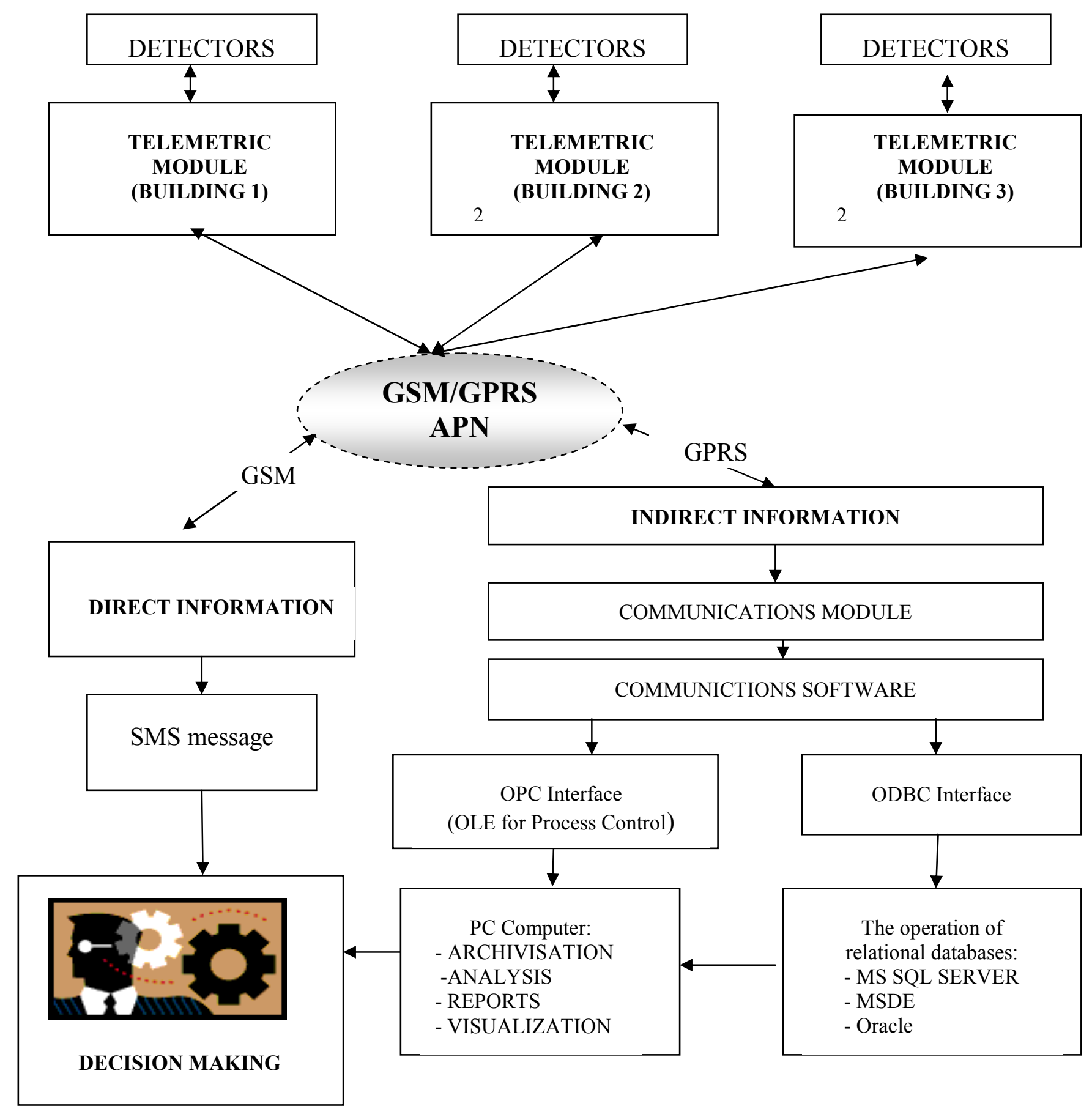

Figure 1. Telemetric system framework 


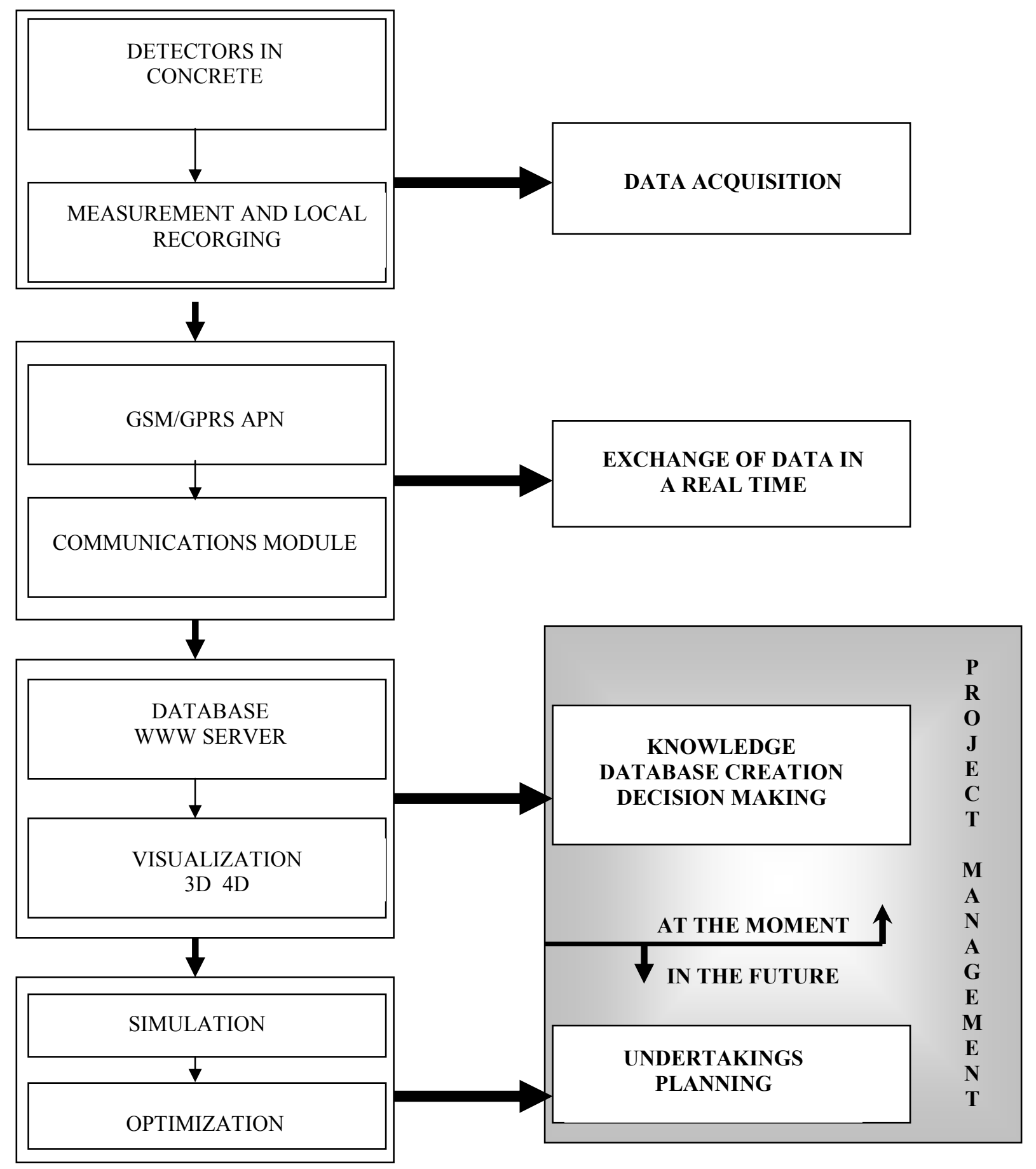

Figure 2. Telemetric system as support project management 


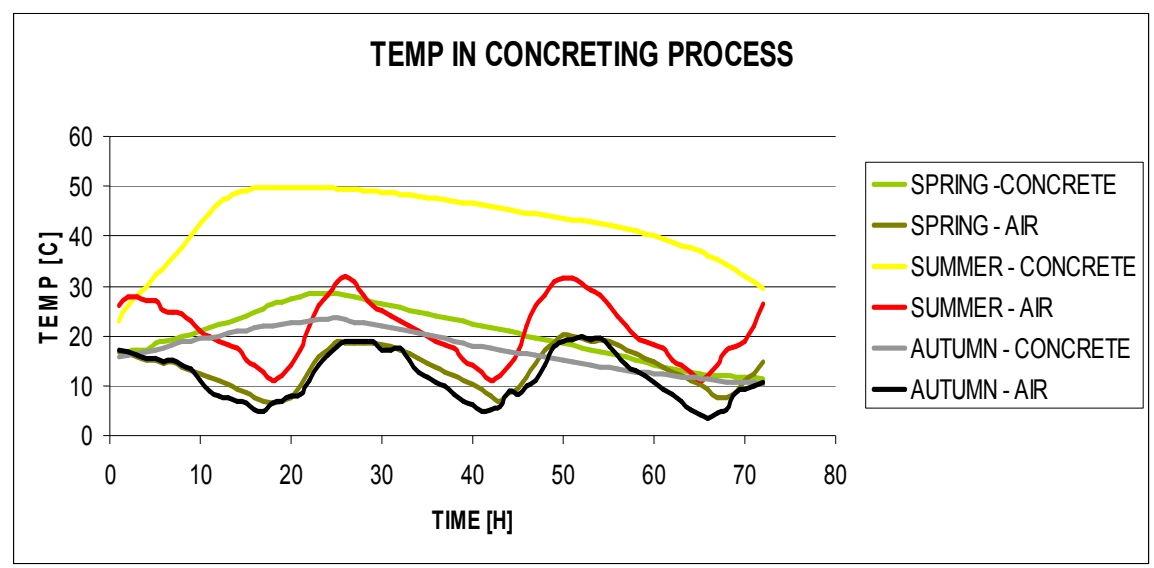

Figure 3. Temperatures distribution in the beginning phase of concrete setting depending on the season

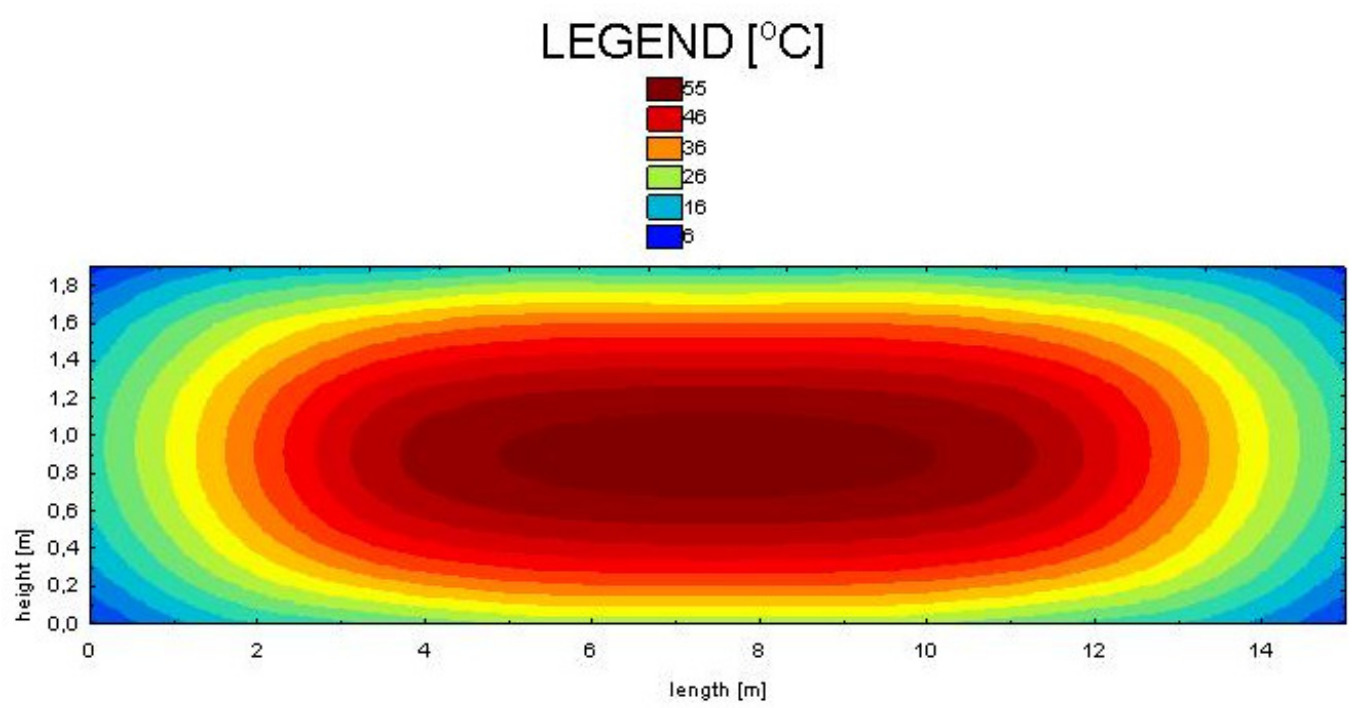

Figure 4. Temperatures distribution in the beginning phase of concrete setting in a concrete block a vertical section 


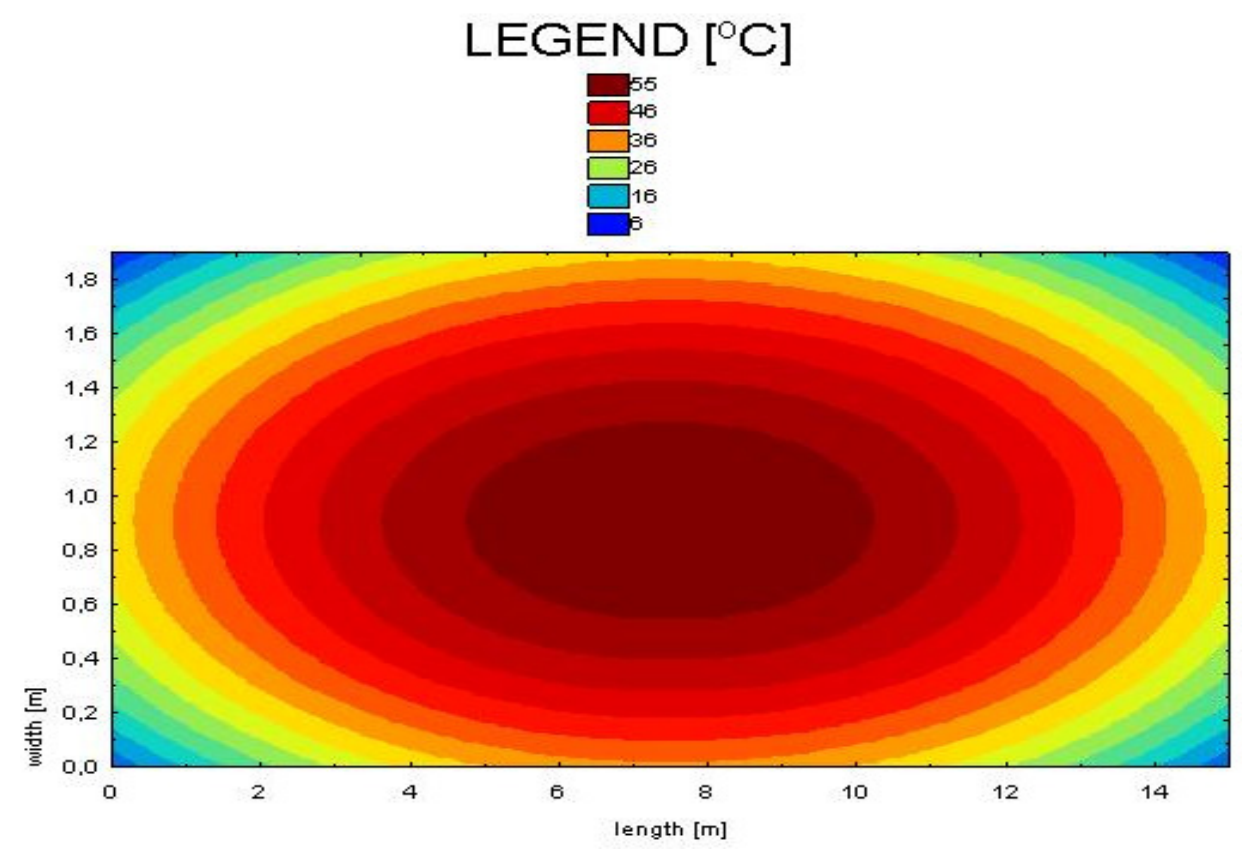

Figure 5. Temperatures distribution in the beginning phase of concrete setting in a concrete block a horizontal section

\section{SUMMARY CONCLUSION}

The conducted research and obtained results confirm usefulness of the above mentioned system to monitor the process of concrete maturing. At present the research on the choice of a device to simulation and optimization of the process of concrete maturing in the beginning phase is being conducted. The device is indispensable to create a comprehensive advisory the use of such easily accessible and popular devices as GSM communication and Internet web gives a great possibility to use it as the advisory system and its further development of this technology. The creation of the comprehensive system will enable to use the research results and measurement in designing and implementing practice which in turn will ensure the high quality of the elements made of concrete.

\section{REFERENCES}

[1] Kapliński, O. (2007) Methods and models of research in construction project engineering, Warszawa.

[2] Zavadskas E.K., Kaklauskas A., Lepkova N., Gikys M. and Banaitis, A. (2002) Web-Based Simulation System for Facilities Management, ACsS'02-SCM conference, Poland, 515-521.

[3] Flexible telemetric system GPRS, Magazine Telemetron 01/08.

[4] D’Obyrn, M. Telemetric system, M.Bulletin Automation 03/07. 